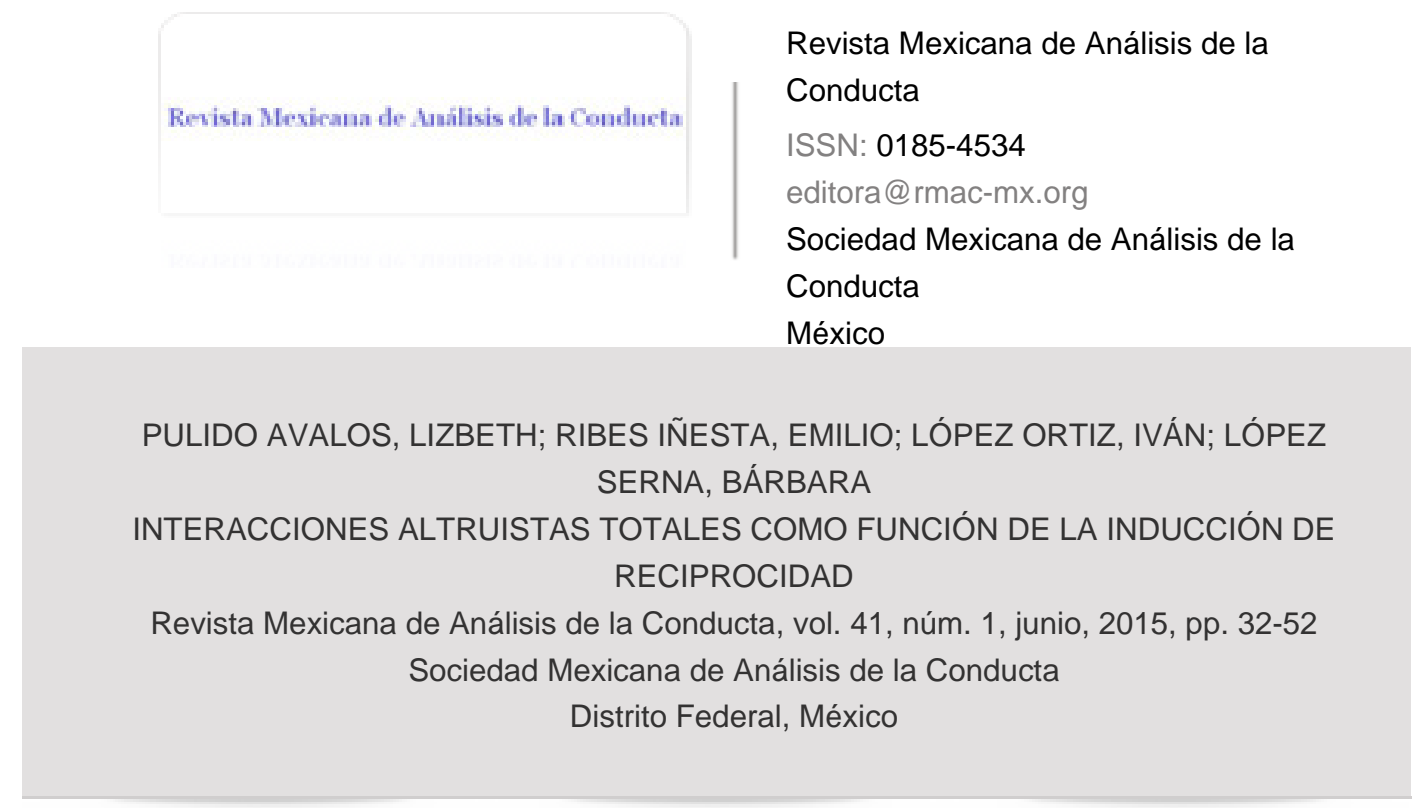

Disponible en: http://www.redalyc.org/articulo.oa?id=59338802002

Cómo citar el artículo

- Número completo

- Más información del artículo

- Página de la revista en redalyc.org

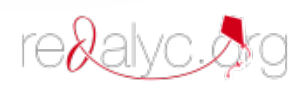

Sistema de Información Científica

Red de Revistas Científicas de América Latina, el Caribe, España y Portugal Proyecto académico sin fines de lucro, desarrollado bajo la iniciativa de acceso abierto 


\title{
INTERACCIONES ALTRUISTAS TOTALES COMO FUNCIÓN DE LA INDUCCIÓN DE RECIPROCIDAD
}

\author{
TOTAL ALTRUISTIC INTERACTIONS AS A FUNCTION OF \\ RECIPROCITY INDUCTION
}

\section{LIZBETH PULIDO AVALOS, EMILIO RIBES IÑESTA, IVÁN LÓPEZ ORTIZ Y BÁRBARA LÓPEZ SERNA UNIVERSIDAD VERACRUZANA, MÉXICO}

\begin{abstract}
Resumen
Se realizó un experimento para evaluar el efecto de la inducción porcentual de reciprocidad por parte de un confederado en la elección entre contingencias no compartidas o contingencias compartidas de altruismo total. La situación experimental consistió en la resolución de rompecabezas virtuales en la pantalla de dos computadoras interconectadas en red. Ocho estudiantes universitarios fueron asignados a ocho díadas experimentales (integradas por un participante y un confederado). En las condiciones experimentales, cada uno de los participantes de la díada pudo observar la ejecución de su compañero y colocar piezas tanto en su rompecabezas (propio) como en el de su compañero (remoto). Todas las díadas fueron expuestas a condiciones experimentales sucesivas en las cuales el porcentaje de piezas colocadas en el rompecabezas remoto por parte del confederado fue de 0, 25, 50, 75 y $100 \%$ para el Grupo 1 y de entre 100, 75, 50, 25 y 0\% para el Grupo 2. Los datos obtenidos mues-

Lizbeth Pulido Avalos, Emilio Ribes Iñesta, Iván López Ortiz y Bárbara López Serna, Universidad Veracruzana.

Se extiende un profundo agradecimiento al Consejo Nacional de Ciencia y Tecnología por las becas otorgadas como ayudantes de investigación a Iván López Ortiz con número de expediente 10399 y Bárbara López Serna con número de expediente 10400, quienes participaron como confederados y asistentes en la aplicación del presente experimento, así como en el análisis de los datos y la revisión del documento final. Los dos primeros autores participaron, además de lo anterior, en la preparación y redacción del documento.

Datos de contacto: Dirigir correspondencia a la primera autora al Centro de Estudios e Investigaciones en Conocimiento y Aprendizaje Humano, Av. Orizaba \# 203, Fraccionamiento Veracruz, C.P. 91020, Xalapa, Veracruz, México. Correo electrónico: Ipulido@uv.mx
\end{abstract}


tran que la conducta altruista total de los participantes fue una función de las piezas que los confederados colocaron en el rompecabezas remoto. Los resultados son discutidos en términos de los tipos de reciprocidad involucrados en todas las relaciones sociales de intercambio.

Palabras clave: altruismo total, reciprocidad, confederado, contingencias sociales

\begin{abstract}
An experiment was conducted to assess the effects of the percentage of induced reciprocity by a confederate on the total altruistic behavior of a peer. The experimental situation consisted of solving puzzles on two synchronized computer screens. The participants were eight college students, who were assigned to eight dyads (each dyad consisting of one participant and one confederate). All dyads were exposed to successive experimental conditions in which the percentage of pieces placed in the remote puzzle by the confederate was varied from 0 to $100 \%$. Half of the dyads was assigned to Group 1 and was exposed to an increasing order (0, 25, 50, 75, and 100 $\%$ ) and the other half was assigned to Group 2 and was exposed to a decreasing order $(100,75,50,25$, and $0 \%)$. Results showed that altruistic behavior was a function of the percentage of remote placements by the confederates. Results are discussed in terms of the kinds of reciprocity involved in all exchange social relations.
\end{abstract}

Keywords: total altruism, reciprocity, confederate, social contingencies

El Diccionario de la Real Academia Española (22 edición) define al término altruismo como la "diligencia en procurar el bien ajeno aun a costa del propio". Post (2002) definió a una persona altruista como "...alguien que hace algo por el otro y por el bien del otro en lugar de hacerlo como un medio para promoverse a sí mismo o para sentirse bien..." (p. 53). Oliner (2002) afirmó que un comportamiento es altruista siempre y cuando: 1) sea dirigido a ayudar a alguien más, 2) involucre riesgo o sacrificio para el actor, 3) no sea acompañado por ninguna recompensa externa y 4) sea voluntario.

Históricamente, diversos corpus conceptuales han intentado dar cuenta del altruismo. Con fines analíticos, pueden ser divididos en cuatro grandes rubros: a) aquellos que afirman que las personas son altruistas por naturaleza (Ashley, discípulo de Locke; Rousseau, 1762/2005); b) aquellos que afirman que las personas son altruistas en función de una norma interiorizada que así se los dicta (Kant, 1785/2006); c) aquellos que afirman que las personas son muy raramente altruistas y que, cuando lo son, es motivado por sentimientos egoístas de autosatisfacción (Hobbes, 1651/2006) y d) aquellos que afirman que las personas son altruistas únicamente con sus consanguíneos (Huxley, 1898; Sahlins, 1972). 
Durante más de un siglo, la masa crítica de las disquisiciones formales en torno al altruismo se concentró en el debate entre la importancia o la futilidad de las relaciones consanguíneas para su emergencia y mantenimiento (Dugatkin, 2006). Mientras que Huxley (1898) sostuvo que entre los organismos impera la lucha por la existencia y que las conductas altruistas son excepcionales e infaltablemente ligadas a la consanguineidad, Kropotkin (1902) postuló, a partir de algunas observaciones naturalistas realizadas en Siberia, que las relaciones entre organismos se soportan en la ayuda mutua, misma que trasciende con creces a la consanguineidad y se da al interior y entre las especies, pues, sin la ayuda mutua, la supervivencia sería improbable.

El grueso de los tratamientos teóricos y/o experimentales so pretexto del estudio de la conducta altruista desde la psicología se publicaron hasta entrados los años 60`s, siendo la investigación de Darley y Latané (1968) la más influyente de la época. Dichos autores diseñaron una serie de experimentos a fin de estimar el grado de participación de observadores casuales en una situación de emergencia. Encontraron, groso modo, que a mayor número de observadores, menor incidencia de conductas altruistas. Los autores concluyeron que los grupos numerosos diluyen la responsabilidad individual de asistir a otros.

Una gran parte de los trabajos tradicionales dedicados al análisis del altruismo desde la psicología fueron aglutinados, pese a su disparidad, al interior de una de sus ramas incipientes, la llamada psicología social. Desde ahí, se postularon teorías inspiradas por disciplinas como la sociología, la economía o la política (e.g., Abelson et al., 1968; Blau, 1964; Thibaut \& Kelley, 1959), y otras más basadas en extensiones de modelos cognoscitivos (e.g., Bandura, 1977; Festinger, 1957; Secord \& Backman, 1974). Por ejemplo, desde la teoría del intercambio social (e.g., Befu, 1977; Blau, 1964; Cook \& Emerson, 1978; Gouldner, 1960; Homans, 1958, 1961; Thibaut \& Kelley, 1959) se propuso que la conducta altruista, así como otras conductas sociales, se explica por la búsqueda mancomunada de la minimización de los costos y la maximización de las recompensas externas (reconocimiento social) o internas (reducción de la ansiedad o autosatisfacción).

Otra postura influyente dentro del área fue la del altruismo recíproco (Trivers, 1971). Según Bowles (2004), el altruismo recíproco puede definirse como aquellas "acciones que confieren un beneficio a otros con un coste para sí mismo pero con la perspectiva de un beneficio recíproco subsiguiente suficiente para cubrir costos" (p. 441). De acuerdo con Trivers (1971) el altruismo recíproco emerge: 1 ) cuando hay numerosas situaciones altruistas, 2) cuando determinado altruista actúa repetidamente con el mismo pequeño conjunto de individuos, y 3) cuando pares de altruistas están expuestos simétricamente a situaciones altruistas. Desde esta postura, la relación costo/be- 
neficio explica la conducta altruista recíproca, pues se supone que el costo del altruismo es compensado con creces por las retribuciones recíprocas asociadas a éste.

Esta perspectiva la retomó Axelrod (1984, 2003), quien, desde la teoría de juegos, una de las posturas más influyentes para el estudio de la conducta altruista en nuestros días (Camerer, 2003; Markis \& Siciliani, 2013), explicó las relaciones altruistas recíprocas a través del argumento de la maximización del beneficio mutuo como el único mecanismo que garantiza la manutención de relaciones de tal tipo entre individuos "racionales". La conducta altruista ha sido definida desde aquí como una serie de actos costosos que confieren beneficios económicos a otros individuos (Fehr \& Fischbacher, 2003; Locey, Safin, \& Rachlin, 2013).

Por su parte, desde el paradigma operante del análisis experimental de la conducta se sostiene que es plausible estudiar la conducta altruista como una conducta operante más, esto es, a través de la triple relación de contingencia. La conducta altruista es definida como aquella conducta que produce el reforzamiento para otros individuos pero no para sí mismo (Boren, 1966; Hake, Olvera, \& Bell, 1975; Hake \& Vukelich, 1972; Schmitt \& Marwell, 1968, 1971a, 1971b; Skinner, 1953). En el estudio prototípico de Weiner (1977), por ejemplo, responder de manera altruista se definió como "dar una respuesta que podía o no producir consecuencias adversas de reforzamiento al participante" (p. 516). Al respecto Skinner (1978) señaló que "aprendemos a ayudar o a hacer el bien... debido a las consecuencias derivadas de ello. Algunas veces ayudamos porque la incapacidad de los demás nos resulta aversiva. Ayudamos a los que nos ayudan, y dejamos de ayudarlos cuando dejan de hacerlo" (p. 33).

Tal y como lo conciben las diversas posturas antes desarrolladas, el altruismo no sería más que una conducta egoísta intermediada y compartida o una forma de reciprocidad interesada (Flace \& Hegselman, 1999). Para Schuster y Perelberg (2004) el estudio de las dimensiones sociales como la historia de trabajo conjunto, la familiaridad con el otro o la jerarquía social es soslayado por este tipo de explicaciones económico-utilitarias. Además, posturas como las anteriores no son capaces de explicar los resultados obtenidos en diversas investigaciones en donde los individuos se comportan de manera altruista aún y cuando este no implique maximización y, en algunos casos, sí menoscabo de sus bienes o ganancias (i.e. juego del ultimátum en Andersen, Ertaç, \& Gneezy, 2011; Güth, Schmittberger, \& Schwarze, 1982; Rand, Tarnita, Ohtsuki, \& Nowak, 2012). Ribes, Rangel y López (2008a) afirmaron que:

En la medida en que las relaciones sociales entre individuos dependen de factores extraindividuales, es incorrecto suponer, como lo hacen las diversas aproximaciones psicológicas, que dichas relaciones ocurren siempre como universales, indistintamente de la estructura institucional de 
cada tipo de formación social. Es incorrecto suponer que la ocurrencia de distintas y variadas formas de comportamiento en las relaciones de intercambio, como son el altruismo, la competencia, la mal llamada cooperación y otras más, dependen exclusivamente de variables especificables en un nivel conductual, independientemente de la historia social y de las funciones institucionales que desempeñan en la vida real. Es incorrecto también suponer que las variables "económicas" relacionadas a la conducta son universales y que reflejan procesos individuales, biológicos y/o psicológicos (pp. 49-50).

Al separarse de las posturas centradas únicamente en factores económico-utilitarios, Ribes y Pulido (2015) terminaron por sostener que las relaciones de reciprocidad constituyen el antecedente y el fondo necesario para la emergencia de relaciones de intercambio tales como el altruismo. De acuerdo con los autores, todas las relaciones de intercambio se originan en alguna forma de reciprocidad. Diferentes evidencias experimentales apoyan estas afirmaciones. Dreman y Greenbaum (1973) condujeron un experimento con niños en edad preescolar como participantes experimentales a fin de explorar la relación entre el altruismo y la reciprocidad. Encontraron, entre otras cosas, que las condiciones de reciprocidad favorecieron la emisión de conductas de donación altruista en comparación con aquellas de no reciprocidad. En esta misma línea, Marcus y Jenny (1977) encontraron que un grupo de niños respondían de manera recíproca ante las conductas de ayuda altruista emitidas por sus pares.

Los asertos generales de Ribes, Rangel y López (2008a) y Ribes y Pulido (2015) antes aludidos se enmarcan dentro de la propuesta teórico-metodológica general planteada por Ribes (2001) para el análisis de las dimensiones funcionales de la conducta social desde una perspectiva interconductual (véase Kantor, 1959, 1980; Ribes \& López, 1985). En ésta, se distinguen tres dimensiones funcionales del medio de contacto convencional constituido por las instituciones como sistemas de prácticas compartidas. El medio de contacto convencional, como formación social, se fundamenta en la división social del trabajo y el diferimiento en tiempo y espacio del intercambio de bienes y servicios posible sólo a través del lenguaje. Las dimensiones funcionales de todo medio de contacto convencional son el poder, el intercambio y la sanción (Ribes, 2001; Ribes, 2010; Ribes \& Pulido, 2015; Ribes, et al., 2008a).

El presente estudio se inserta en el ámbito de las contingencias de intercambio, entre las que destacan como casos representativos el altruismo parcial y el altruismo total. Mientras que el altruismo parcial ha sido definido como dar y recibir, el altruismo total ha sido definido como dar sin recibir (Ribes 2001; Ribes, 2010; Ribes, et al., 2008a). La propuesta experimental general empleada desde esta tradición ha buscado evaluar, entre otras cosas, los factores que intervienen en la elección entre contingencias individuales o no compartidas y contingencias compartidas de altruismo (total o parcial). 
Para ello, se ha empleado una tarea de resolución de rompecabezas en una situación diádica en la que se presentan dos alternativas de respuesta de manera concurrente: una individual o no compartida (rompecabezas propio) y una social o compartida de altruismo parcial o total (rompecabezas del compañero). Mientras que en el procedimiento empleado en la situación de altruismo parcial las piezas colocadas por el participante en el rompecabezas de su compañero generan puntos iguales para él y para su compañero, en la situación de altruismo total las piezas colocadas por el participante en el rompecabezas de su compañero generan puntos únicamente para éste último.

Los resultados de los estudios conducidos desde esta tradición han mostrado que en situaciones de elección libre entre contingencias no compartidas y contingencias compartidas de altruismo parcial o total, los participantes responden de manera casi exclusiva bajo contingencias no compartidas, a pesar de la reducción significativa de sus ganancias (Ribes \& Rangel, 2002; Ribes, Rangel, Carbajal \& Peña, 2003a; Ribes, Rangel, Casillas et al., 2003b; Ribes, Rangel, Juárez et al., 2003c).

Una de las notables excepciones a este hallazgo general fue la reportada en los estudios de Ribes, Rangel, Ramírez et al. (2008b), Ribes et al. (2010) y Rangel, Pulido, Ávila, Ordoñez y Ribes (en prensa) en donde se expuso a díadas de adultos (conformadas por un participante y un confederado) a un procedimiento de inducción de reciprocidad a fin de estimar su efecto en la elección de contingencias no compartidas y/o compartidas de altruismo parcial. Se encontró que las respuestas dadas bajo la contingencia no compartida o compartida de altruismo parcial por parte de los participantes estaban en función de las respuestas de los confederados, a punto tal que las respuestas de los participantes en tal o cual alternativa se correlacionaron casi en proporción 1:1 con la de los confederados. El confederado indujo indiferencia o altruismo parcial recíprocos cuando respondió, respectivamente, bajo la alternativa no compartida o compartida de altruismo parcial ante una respuesta dada bajo la misma alternativa por parte del participante y el participante, a su vez, respondió secundado la elección del confederado.

Sin embargo, resta explorar si estos resultados pueden replicarse, en lo general, cuando la contingencia compartida implicada no es de altruismo parcial sino total. Lo anterior es relevante puesto que permitiría determinar si la inducción de reciprocidad es efectiva para promover respuestas bajo contingencias compartidas aun y cuando estas respuestas no impliquen ganancias para el participante que las emite.

El presente trabajo pretendió explorar si la inducción de conducta recíproca por parte de un confederado es suficiente para el establecimiento de interacciones indiferentes y altruistas totales. Para ello, se replicó parcialmente el procedimiento empleado en Ribes, et al. (2010) de inducción porcentual de reciprocidad ascendente y descendente por parte de un confederado, procedimiento efectivo para establecer interacciones altruistas parciales e indiferentes ${ }_{i}$ Es la inducción porcentual de reci- 
procidad igualmente efectiva para el establecimiento de interacciones indiferentes $y$, sobre todo, totalmente altruistas? ¿Se pueden desarrollar respuestas totalmente altruistas sin que el participante esté obligado a darlas? A fin de dar una respuesta tentativa a tales interrogantes se planeó el experimento que se describe a continuación.

\section{Método}

\section{Participantes}

Participaron ocho estudiantes universitarios, cuatro hombres y cuatro mujeres, de entre los 20 y los 29 años de edad, a cambio de discos musicales. Los participantes fueron asignados al azar en ocho díadas y tuvieron como pareja experimental a un confederado. Los confederados fueron un hombre y una mujer, ambos estudiantes universitarios, que fueron entrenados especialmente para este experimento.

\section{Aparatos y situación experimental}

Se utilizaron dos equipos de cómputo con procesador Pentium Dual Core, interconectados y sincronizados, con monitor cromático, teclado y controles de juego para responder. Las instrucciones y la tarea experimental (armado de rompecabezas) se presentaron en la pantalla del monitor. Las respuestas de los participantes fueron registradas automáticamente por el programa de cómputo, que fue diseñado en el lenguaje Blitz Max y ejecutado en el sistema Windows 7 . Los datos se analizaron con el programa IBM SPSS Statistics versión 20.0 y se graficaron con el programa Sigma Plot versión 12.0.

Las sesiones se llevaron a cabo en el Centro de Estudios e Investigaciones en Conocimiento y Aprendizaje Humano durante cinco días consecutivos. El primer día se realizaron dos sesiones de exposición a la tarea y tres experimentales y, a partir del segundo día, los sujetos se expusieron a tres sesiones experimentales diarias. Los participantes trabajaron en cubículos separados, sonoamortiguados y libres de distractores.

\section{Procedimiento}

Para el presente estudio se utilizó un diseño experimental intrasujeto con comparación entre sujetos bajo la misma y bajo distintas condiciones experimentales. Los participantes se distribuyeron al azar en dos grupos, cada uno integrado por cuatro díadas (ver Tabla 1). Todos los participantes tuvieron como compañero de díada a un confederado que respondió de forma planeada conforme al diseño experimental. 
Tabla 1

Diseño Experimental empleado en el estudio

\begin{tabular}{|c|c|c|c|c|c|c|c|}
\hline \multirow{2}{*}{ Grupo } & \multirow{2}{*}{$\begin{array}{l}\text { Sesión de } \\
\text { exposición A }\end{array}$} & \multirow{2}{*}{$\begin{array}{l}\text { Sesión de } \\
\text { exposición B }\end{array}$} & \multicolumn{5}{|c|}{ Fase } \\
\hline & & & 1 & 2 & 3 & 4 & 5 \\
\hline $\begin{array}{c}1 \\
(n=4 \\
\text { díadas })\end{array}$ & $\begin{array}{c}\text { Sesión de } \\
\text { exposición } \\
\text { A (ejecución } \\
\text { independiente) }\end{array}$ & $\begin{array}{l}\text { Sesión de } \\
\text { exposición } \\
\text { B (ejecución } \\
\text { simultánea) }\end{array}$ & $0 \%$ & $25 \%$ & $50 \%$ & $75 \%$ & $100 \%$ \\
\hline $\begin{array}{c}2 \\
(n=4 \\
\text { díadas })\end{array}$ & $\begin{array}{c}\text { Sesión de } \\
\text { exposición } \\
\text { A (ejecución } \\
\text { independiente) }\end{array}$ & $\begin{array}{l}\text { Sesión de } \\
\text { exposición } \\
\text { B (ejecución } \\
\text { simultánea) }\end{array}$ & $100 \%$ & $75 \%$ & $50 \%$ & $25 \%$ & $0 \%$ \\
\hline Sesiones & 1 & 1 & 3 & 3 & 3 & 3 & 3 \\
\hline Días & $1^{\circ}$ & $1^{\circ}$ & $1^{\circ}$ & $2^{\circ}$ & $3^{\circ}$ & $4^{\circ}$ & $5^{\circ}$ \\
\hline
\end{tabular}

Antes de iniciar con la tarea experimental, se entregó a los participantes un documento de consentimiento informado en donde se les notificaba sobre su rol en el estudio, sus derechos a lo largo de éste, los requerimientos implicados para su consecución y la información de contacto necesaria para cualquier consulta posterior. Los participantes leyeron, completaron y firmaron adecuadamente el documento y al finalizar el primer día se les entregó una copia del mismo.

Se utilizó una tarea de resolución de rompecabezas virtuales como procedimiento. Se presentó un rompecabezas distinto para cada sesión experimental. Los participantes pudieron ver la imagen del rompecabezas a armar durante un minuto, posteriormente ésta se dividió en 50 piezas que se distribuyeron al azar y se desplegó un cursor operado por el control de juego que sirvió a los participantes para la selección y colocación de las piezas del rompecabezas. En la pantalla aparecieron uno o dos rompecabezas dependiendo de la condición experimental en turno. La Figura 1 muestra el arreglo de la pantalla presentada a los participantes durante las fases experimentales, mientras que la Figura 2 muestra ejemplos de las imágenes de los rompecabezas utilizados en este estudio. 


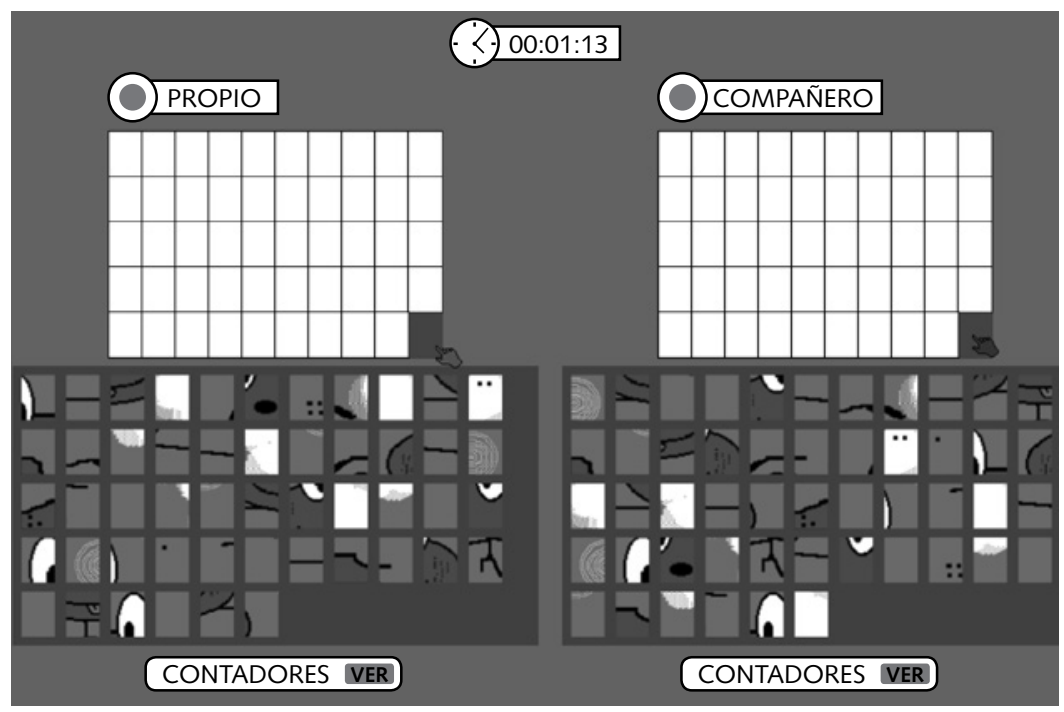

Figura 1. Arreglo de la pantalla de la computadora durante las distintas fases experimentales y la sesión de exposición a la tarea B. Durante la sesión de exposición a la tarea A, apareció solamente un rompecabezas en pantalla. No se presentaron los puntajes durante las sesiones de exposición a la tarea.
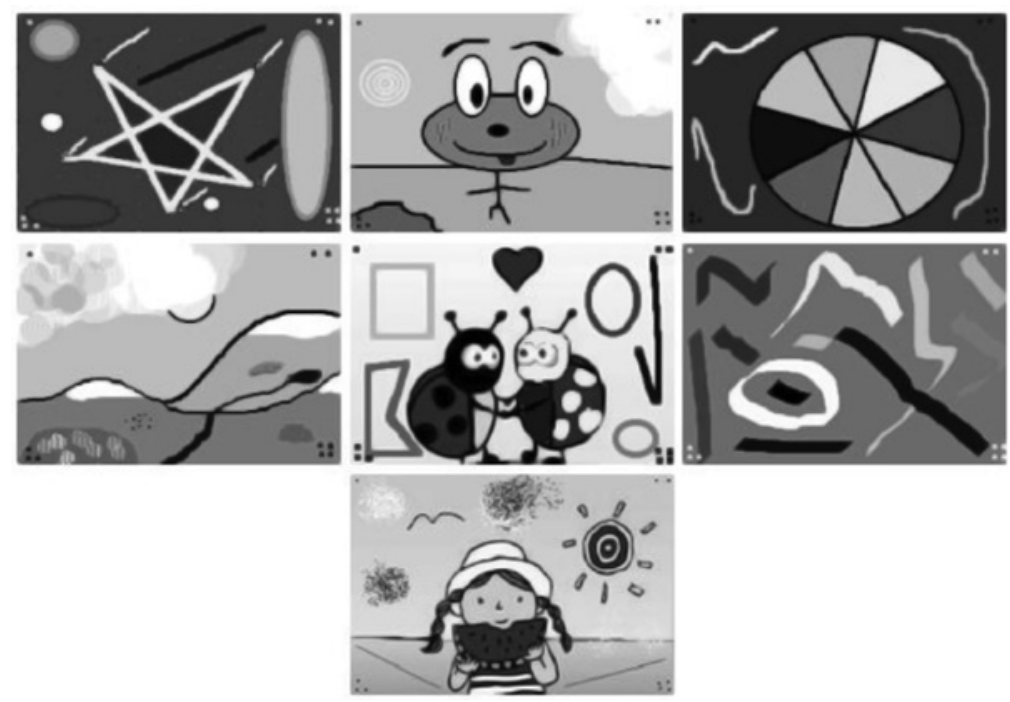

Figura 2. Ejemplos de las imágenes de los rompecabezas de 50 piezas utilizados en este experimento. 


\section{Sesión de exposición a la tarea $\mathrm{A}$}

Ejecución independiente sin retroalimentación. En esta condición apareció un solo rompecabezas en pantalla. Cada participante resolvió su rompecabezas de manera independiente y el tiempo para hacerlo fue ilimitado. Los participantes no recibieron puntos por la colocación de las piezas, por lo que no se presentó ningún contador.

\section{Sesión de exposición a la tarea B}

Ejecución simultánea sin retroalimentación. En esta condición aparecieron dos rompecabezas en la pantalla. En el lado izquierdo apareció un rompecabezas denominado "Propio" y en el lado derecho otro rompecabezas denominado "Del compañero". El participante sólo pudo colocar las piezas de su propio rompecabezas, mientras que el otro rompecabezas fue resuelto por el confederado quien colocó una pieza de manera correcta cada 5 segundos. El tiempo de resolución fue ilimitado. Cuando el participante terminó de armar el rompecabezas "propio" se activó el botón llamado "FIN" el cual podía presionar y con ello terminar la tarea para él y su compañero (en este caso el confederado quien nunca presionó el botón "FIN"). Ni los participantes ni los confederados recibieron puntos por la colocación de las piezas, por lo que no se presentó ningún contador.

\section{Sesiones experimentales $0 \%$ a $100 \%-100 \%$ a $0 \%$}

En esta condición aparecieron dos rompecabezas en la pantalla. En el lado izquierdo apareció un rompecabezas denominado "Propio" y en el lado derecho otro rompecabezas denominado "Del compañero". El participante y el confederado pudieron responder en cualquiera de los dos rompecabezas que aparecieron en la pantalla (el confederado respondió de acuerdo al diseño experimental). Se presentaron cinco fases experimentales (una por día): una fase de $0 \%$ de reciprocidad, una de $25 \%$, una de $50 \%$, una de $75 \%$ y una de $100 \%$. El porcentaje de reciprocidad estuvo en función del número de piezas posible a colocar (50 piezas). El Grupo 1 se expuso a una secuencia de $0,25,50,75$ y $100 \%$ de respuestas de reciprocidad por parte del confederado. El Grupo 2 se expuso a una secuencia 100, 75, 50, 25 y 0\% de respuestas de reciprocidad por parte del confederado.

En la fase de 100\%, el confederado colocó todas las piezas posibles en el rompecabezas del compañero, en un caso "ideal" colocó 50 piezas en ese rompecabezas y no colocó ninguna pieza en su rompecabezas. En la fase de $75 \%$, el confederado trató de colocar 38 piezas en el rompecabezas de su compañero y 13 piezas en su rompecabezas, que equivalía a un $25 \%$ de las piezas posibles a colocar en ese rom- 
pecabezas. En la fase de 50\% el confederado trató de colocar 25 piezas en el rompecabezas del compañero y 25 piezas en el suyo, que equivalía a $50 \%$ de las piezas posibles de colocar en ese rompecabezas. En la fase de $25 \%$ el confederado trató de colocar 13 piezas en el rompecabezas del compañero y 38 piezas en su rompecabezas que equivalía al $75 \%$ de las piezas posibles a colocar en ese rompecabezas; en la fase de $0 \%$ el confederado no colocó ninguna pieza en el rompecabezas del compañero y trató de colocar 50 piezas en su rompecabezas. El confederado respondió según el ritmo de respuesta de cada participante, tratando de colocar una pieza por cada pieza colocada por el participante.

El tiempo para resolver los rompecabezas fue ilimitado. Si un participante de la díada terminaba el rompecabezas denominado "propio" podía presionar el botón llamado "FIN" y terminar la tarea para él y para su compañero (el confederado), también tuvo la opción de no presionarlo y esperar a que el compañero terminara la tarea. Los confederados nunca presionaron el botón "FIN".

Tanto los participantes como los confederados recibieron puntos por cada pieza colocada correctamente. Cuando colocaron una pieza en su propio rompecabezas obtuvieron 10 puntos para sí mismos y no le dieron ningún punto a su compañero. Cuando colocaron una pieza en el rompecabezas del compañero le dieron 10 puntos a su compañero y no obtuvieron ningún punto para ellos mismos. Los puntos acumulados pudieron visualizarse en cualquier momento de la sesión al presionar el botón denominado "VER CONTADORES".

Al finalizar el experimento se les pidió a los participantes que respondieran verbalmente a la siguiente pregunta: ¿Por qué respondiste en el rompecabezas de tu compañero? Esto con el fin de recabar, de manera informal, información adicional que pudiera eventualmente resultar de interés para el estudio.

\section{Resultados}

Las Figuras 3 y 4 muestran el número promedio por fase de piezas colocadas de manera correcta en el rompecabezas local y en el rompecabezas remoto por los participantes y los confederados de los Grupos 1 y 2. Los datos de las sesiones de exposición a la tarea no fueron incluidos, sin embargo, todos los participantes colocaron correctamente todas las piezas del rompecabezas local. Las filas de gráficos denominadas "Local" indican el número promedio por fase de piezas colocadas correctamente en el rompecabezas propio. Las filas de gráficos denominadas "Remoto" indican el número promedio por fase de piezas colocadas correctamente en el rompecabezas del compañero.

La Figura 3 muestra que los participantes de las cuatro díadas del Grupo 1 incrementaron el número de colocaciones correctas de piezas en el rompecabezas remo- 


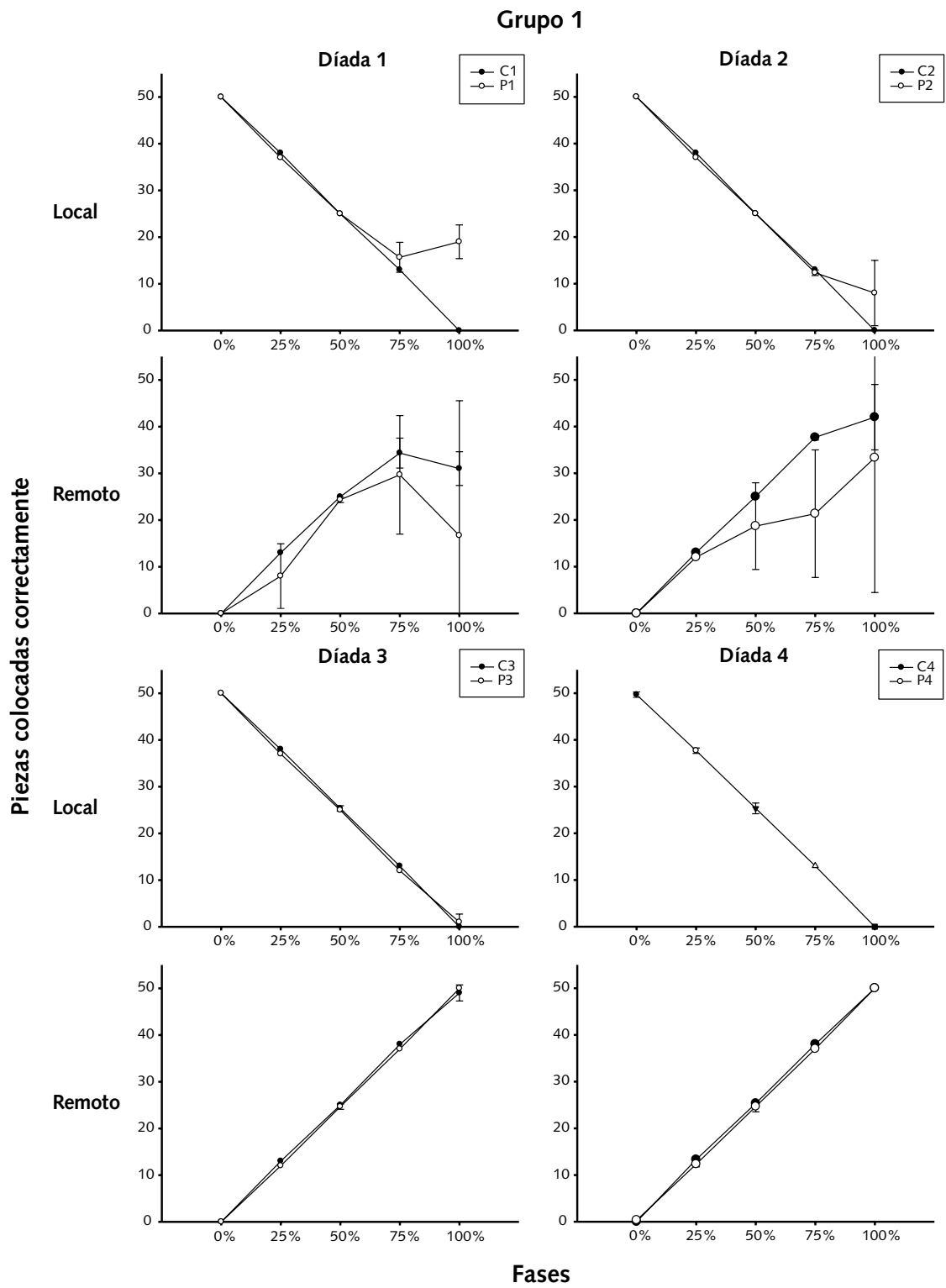

Figura 3. Número promedio por fase de piezas colocadas correctamente por los participantes y los confederados del Grupo 1. Las líneas y círculos representan cada una de las condiciones experimentales. Los círculos blancos corresponden a los participantes (P) mientras que los círculos negros corresponden a los confederados (C). Las barras de error indican las desviaciones estándar de las medias. 


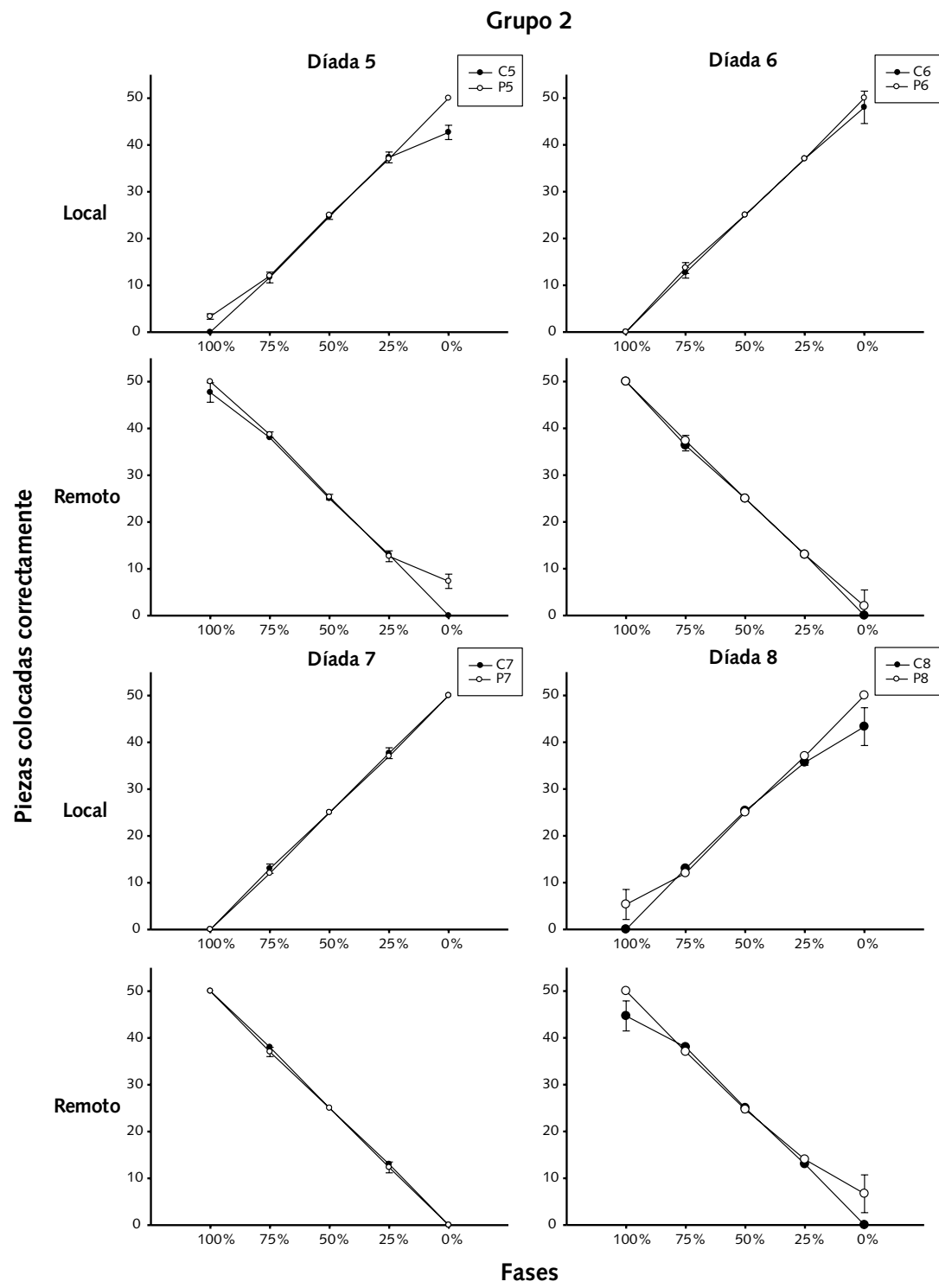

Figura 4. Número promedio por fase de piezas colocadas correctamente por los participantes y los confederados del Grupo 2. Las líneas y círculos representan cada una de las condiciones experimentales. Los círculos blancos corresponden a los participantes (P) mientras que los círculos negros corresponden a los confederados (C). Las barras de error indican las desviaciones estándar de las medias. 
to de acuerdo con el porcentaje ascendente de reciprocidad del confederado en las sucesivas fases experimentales, con excepción del participante 1 en la Fase 5 con $100 \%$ de reciprocidad. El número de colocaciones correctas en el rompecabezas local y en el remoto por los participantes de las díadas 3 y 4 fue prácticamente el mismo que el de las colocaciones en cada uno de los rompecabezas del confederado. Los participantes de las díadas 1 y 2 , en cambio, colocaron menos piezas en el rompecabezas remoto que el confederado (excepto en la Fase 1 de $0 \%$ de reciprocidad). Sólo los participantes de los Díadas 1 y 2 presionaron el botón "FIN" para terminar la sesión experimental antes del armado de los dos rompecabezas. El participante 1 lo hizo en 6 de las 15 sesiones experimentales, mientras que el participante 2 lo hizo en 5 de 15.

La Figura 4 muestra que los participantes de las cuatro díadas del Grupo 2 disminuyeron la colocación de piezas en el rompecabezas remoto en función del porcentaje de reciprocidad decreciente del confederado en las sucesivas fases experimentales. El número colocaciones correctas en el rompecabezas remoto y local por parte de los participantes fue igual o ligeramente más alto que las colocaciones recíprocas de los confederados. Los participantes de las díadas 5 y 8 colocaron más piezas que los confederados en el rompecabezas remoto en las fases 1 y 5 de 0 y $100 \%$ de reciprocidad respectivamente, lo que muestra que estos resultados no son un artefacto del procedimiento tal y como se ha reportado ampliamente en Rangel et al. (en prensa). Los participantes de este grupo nunca presionaron el botón "FIN", por lo que las sesiones experimentales terminaron hasta que se completaron los dos rompecabezas en la pantalla.

\section{Discusión}

En general, los resultados de este estudio sugieren que la conducta altruista total de los participantes se relacionó directamente con el porcentaje de conducta recíproca de los confederados. Los participantes colocaron piezas en el rompecabezas de los confederados en una proporción similar a las colocaciones recíprocas de los confederados en los rompecabezas de los participantes, pese a no recibir retribución alguna por hacerlo.

La conducta de los confederados en efecto indujo reciprocidad en la medida en que los participantes colocaron, en general, un número similar de piezas que las colocadas por el confederado en las sucesivas fases experimentales en los rompecabezas local y remoto. La reciprocidad inducida reguló la cantidad de interacciones totalmente altruistas (respuestas en el rompecabezas remoto), así como recíprocamente indiferentes (respuestas en el rompecabezas local). 
El porcentaje diferencial y transicional de respuestas de los participantes en el rompecabezas remoto y en el local durante las fases experimentales puede ser interpretado, de acuerdo con Rangel et al. (en prensa), como un flujo continuo de reciprocidad entre la indiferencia recíproca (tú respondes en tu rompecabezas, yo respondo en el mío) y la reciprocidad totalmente altruista (tú respondes en mi rompecabezas, yo respondo en el tuyo). Es importante insistir en que las elecciones recíprocas del rompecabezas remoto por parte de los participantes se presentaron a pesar de que éstas no producían puntos para ellos. Los participantes podían colocar sus piezas en los espacios restantes de su propio rompecabezas durante su llenado a lo largo de la sesión. De este modo podrían haber obtenido el total de puntos posibles, sin que los obtuviera el compañero (el confederado). Sin embargo, a pesar de ser opcional, colocaron cuando menos en el rompecabezas remoto el número proporcional de piezas que fueron colocadas en el suyo.

A diferencia de los estudios conducidos por Ribes et al. (2010) y Rangel et al. (en prensa), en los que el efecto de la inducción de reciprocidad en la elección de alternativas compartidas de altruismo parcial no podía escindirse, en sentido estricto, del de las ganancias obtenidas por los participantes por responder bajo dichas alternativas, los datos recabados en este trabajo muestran que aun en ausencia de ganancias por hacerlo, los participantes responden bajo alternativas compartidas de altruismo total siempre y cuando su compañero lo haga también.

Estos resultados sugieren que las interacciones altruistas totales parecen depender de la conducta recíproca del compañero más que de la cantidad de ganancias extrínsecas, como han supuesto diversas posturas económico-utilitarias (e.g. Axelrod, 2003; Rachlin, 2002; Skinner, 1953, 1978; Thibaut \& Kelly, 1959). Los resultados del presente estudio muestran que la reciprocidad puede establecerse, al menos localmente, a partir de respuestas iniciadas e inducidas por un compañero. Cuando los confederados colocaban piezas en el rompecabezas remoto o local los participantes correspondían, generalmente, respondiendo en la misma alternativa. Ambas interacciones, altruistas totales o indiferentes, estuvieron conformadas por episodios locales de elección del confederado y del participante.

En experimentos anteriores (Ribes \& Rangel, 2002; Ribes, Rangel, Carbajal, \& Peña, 2003a; Ribes, Rangel, Casillas et al., 2003b; Ribes, Rangel, Juárez et al., 2003c), se encontró consistentemente que bajo condiciones de elección entre contingencias no compartidas y contingencias compartidas de altruismo parcial o total, los participantes preferían responder bajo contingencias no compartidas (rompecabezas local). La preferencia por la contingencia no compartida fue consistente entre ambos integrantes de las díadas. Este hallazgo podría ser reinterpretado a la luz de los presentes y anteriores resultados (Rangel et al., en prensa; Ribes et al., 2010) en términos del es- 
tablecimiento de interacciones de indiferencia recíproca entre los participantes, del tipo "tú no colocas piezas en mi rompecabezas, yo no coloco piezas en el tuyo".

La comparación de los datos obtenidos en el Grupo 1 y en el Grupo 2, expuestos a una secuencia de inducción de reciprocidad descendente-ascendente, ascendentedescendente respectivamente, sugieren, además, que la historia particular de intercambio se relaciona con la sistematicidad de la reciprocidad altruista o indiferente. Todos los participantes del Grupo 2 respondieron en proporción prácticamente idéntica a la del confederado en los rompecabezas remoto y local en las sucesivas fases experimentales, y nunca presionaron el botón para terminar la sesión experimental anticipadamente. En cambio, dos de los participantes del Grupo 1 (1 y 2) sub-igualaron las respuestas del confederado en el rompecabezas remoto y terminaron anticipadamente algunas sesiones una vez que su rompecabezas estaba terminado.

La historia interpersonal de interacciones recíprocas no se restringe a la conducta que tiene lugar en el episodio de intercambio en la situación de altruismo, sino que involucra además el desarrollo de juicios cualitativos como la confianza (Cox, Sadiraj, \& Sadiraj, 2001; Vuolevi \& Van Lange, 2012), o la reputación (Benabou \& Tirole, 2004), relacionados con variables verbales como los acuerdos, las declaraciones, las descripciones o las expectativas. Se puede atribuir a estos factores que la respuesta de la mayoría de los participantes ante la pregunta ¿Por qué respondiste en el rompecabezas de tu compañero?, realizada al finalizar el experimento a fin de no afectar sus resultados, se resumiera en declaraciones del tipo "Él me estaba ayudando, sería mal visto que yo no hiciera lo mismo" o "confiaba en que él me ayudaría si yo hacía lo mismo".

Estos hallazgos, así como los de otros estudios (Henrich \& Henrich, 2007; Marwell \& Schmitt, 1975; Ostrom \& Walker, 2003; Rangel et al., en prensa; Ribes et al., 2010; Torche \& Valenzuela, 2011) sugieren que el altruismo, así como otras formas de conducta social, involucra el establecimiento de una compleja red de relaciones de reciprocidad, las cuales constituyen el antecedente y el trasfondo necesario para la emergencia de relaciones de intercambio en situaciones de altruismo, así como algunas de poder y sanción (Ribes \& Pulido, 2015). Aunque las relaciones de reciprocidad no son siempre inmediatas, directas, homogéneas y simétricas, las relaciones recíprocas así caracterizadas parecen constituir la base real sobre la cual se construyen los diversos tipos de reciprocidad identificables que, eventualmente, cimentarían relaciones de intercambio diversas caracterizadas por la división social del trabajo (Molm, 2010; Ribes, 2001).

En síntesis, los resultados del presente experimento demuestran que las interacciones altruistas totales así como las de indiferencia se basan en la reciprocidad entre los miembros implicados en la interacción más que en factores relativos a la consangui- 
neidad (e.g. Huxley, 1898; Sahlins, 1972), o la maximización eventual de las ganancias (e.g. Axelrod, 2003; Bowles, 2004; Markis \& Siciliani, 2013).

A partir de los resultados del presente estudio se perfilan nuevas perspectivas de investigación. Éstas incluyen la exploración de diversos parámetros de la reciprocidad simétrica y asimétrica tanto en respuestas como en consecuencias pertinentes para el establecimiento de interacciones totalmente altruistas. Asimismo, resulta fundamental indagar el papel del establecimiento de diferentes tipos de interacción verbal entre los participantes en la emergencia de interacciones altruistas más estables, no necesariamente circunscritas a la situacionalidad.

\section{Referencias}

Abelson, R., Aronson, E., McGuire, W., Newsomb, T., Rosernberg, M., \& Tannenbaum, P. (1968). Theories of cognitive consistency: A sourcebook. New York, NY: Rand McNally.

Axelrod, R. (1984). The Evolution of Cooperation. New York, NY: Basic Books.

Andersen, S., Ertaç, S., \& Gneezy, U. (2011). Stakes matter in ultimatum games. American Economic Review, 101, 3427-3439.

Axelrod, R. (2003). The Complexity of Cooperation: Agent-Based Models of Competition and Collaboration. Princeton, NJ: Princeton University Press.

Bandura, A. (1977). Social learning theory. New Jersey, NJ: Prentice-Hall.

Befu, H. (1977). Social Exchange. Annual Review of Anthropology, 6, 225-281.

Benabou, R., \& Tirole, J. (2004). Incentives and prosocial behavior. Mimeo. Princeton University.

Blau, P. M. (1964). Exchange and power in social life. New York, NY: Wiley.

Boren, J. J. (1966). An Experimental Social Relation between Two Monkeys. Journal of the Experimental Analysis of Behavior, 9, 691-700.

Bowles, S. (2004). Microeconomics: behavior, institutions, and evolution. Princeton, NJ: Princeton University press.

Camerer, C.F. (2003). Behavioral Game Theory: Experiments on strategic interaction. New Jersey, NJ: Princeton University Press.

Cook, K. S., \& Emerson, R. M. (1978). Power, equity and commitment in exchange networks. American Sociological Review, 43, 721-739.

Cox, J. C., Sadiraj, K., \& Sadiraj, V. (2001). Trust, fear, reciprocity and altruism. Mimeo. University of Arizona.

Darley, J. M., \& Latané, B. (1968). Bystander intervention in emergencies: Diffusion of responsibility. Journal of Personality and Social Psychology, 8, 377-383.

Dreman, S. B., \& Greenbaum, C. W. (1973). Altruism or reciprocity: sharing behavior in Israeli kindergarten children. Child Development, 44, 61-68. 
Dugatkin, L. A. (2006). The altruism equation. Seven scientists search for the origins of goodnees. Princeton, NJ: Princenton university Press.

Fehr, E., \& Fischbacher, U. (2003). The nature of human altruism. Nature, 425, 785-791.

Festinger, L. (1957). A theory of cognitive dissonance. Stanford, CA: Stanford University Press.

Flace, A., \& Hegselmann, R. (1999). Altruism versus self-interest in social support computer simulations of cooperation and partner selection in support networks. En: E.J. Lawler (Series Ed.) \& S. R. Thye, E. J. Lawler, M. W, Macy, \& H.A. Walker (Vol. Eds.), Advances in group processes, Vol. 16, (pp. 61-97). Stanford, Connecticut: JAI Press Inc.

Gouldner, A. W. (1960). The norm of reciprocity: A preliminary stamen. American Sociological Review, 25, 161-178.

Güth, W., Schmittberger, R., \& Schwarze, B. (1982). An experimental analysis of ultimatum bargaining. Journal of Economic Behavior and Organization, 3(4), 367-388.

Hake, D. F., Olvera, D., \& Bell, J. C. (1975). Switching from Competition to Sharing or Cooperation at Large Response Requirements: Competition Requires more Responding. Journal of the Experimental Analysis of Behavior, 24, 343-354.

Hake, D., \& Vukelich, R. (1972). A Classification and Review of Cooperation Procedures. Journal of the Experimental Analysis of Behavior, 18, 333-343.

Henrich, N., \& Henrich, J. (2007). Why humans cooperate. A cultural and evolutionary explanation. New York, NY: Oxford University Press.

Hobbes, T. (1651/2006). Leviatán o la materia, forma y poder de una república, eclesiástica y civil. México: Fondo de Cultura Económica.

Homans, G. C. (1958). Social behavior as exchange. American Journal of Sociology, 63, 597-606.

Homans, G. C. (1961). Social behavior: Its elementary forms. New York, NY: Harcourt Brace \& Jovanovich.

Huxley (1898). Struggle for existence and its bearing upon man. Nineteenth Century, 23, $161-180$.

Kant, I. (1785/2006). La metafísica de las costumbres. Madrid, España: Alianza.

Kantor, J. R. (1959). Interbehavioral Psychology. Chicago, IL: Principia Press.

Kantor, J. R. (1980). Manifiesto of interbehavioral psychology. Revista Mexicana de Análisis de la Conducta, 6, 117-128.

Kropotkin, P. (1902). Mutual aid. A factor of evolution. London, England: William Heinemann.

Locey, M., Safin, V., \& Rachlin, H. (2013). Social discounting and the prisoner's dilemma game. Journal of the Experimentañ Analysis of Behavior, 99(1), 85-97.

Marcus, R. F., \& Jenny, B. (1977). A naturalistic study of reciprocity in the helping behavior of young children. Alberta Journal of Educational Research, 23, 195-206. 
Markis, M., \& Siciliani, L. (2013). Optimal incentive schemes for altruistic providers. Journal of Public Economic Theory, 15(5), . 675-699.

Marwell, G., \& Schmitt, D. (1975). Cooperation: An experimental analysis. New York, NY: Academic Press.

Molm, L. (2010). The Structure of Reciprocity. Social Psychology Quarterly, 73(2), 119-131.

Oliner, S. (2012) Extraordinary acts of ordinary people: Faces of heroism and altruism. En: S. Post, L. Underwood, J. Schloss, \& W. Hurlbut (Eds.). Altruism and altruistic love: Science, philosophy and religion in dialogue (pp. 123-139). New York, NY: Oxford University Press.

Ostrom, E., \& Walker, J. (2003). Trust and reciprocity: Interdisciplinary lessons from experimental research. New York, NY: Russell Sage Foundation.

Post, S. (2002). The tradition of agape. En: S. Post, L. Underwood, J. Schloss, W. Hurlbut, W. (Eds.). Altruism and altruistic love: Science, philosophy and religion in dialogue (pp. 51-64). New York, NY: Oxford University Press.

Rachlin, H. (2002). Altruism and selfishness. Behavioural and Brain Sciences, 25, 2, 239-250.

Rand, D. G., Tarnita, C. E., Ohtsuki, H., \& Nowak M. A. (2013). Evolution of fairness in the one-shot anonymous ultimatum game. Procedings of the National Academy of Sciences of the United States of America, 110(7), 2581-2586.

Rangel, N., Pulido, L., Ávila, A., Ordoñez, S., \& Ribes, E. (en prensa). Partial altruistic interactions as a function of reciprocity induction and written declarations. European Journal of Behavior Analysis.

Real Academia Española. (2001). Diccionario de la lengua española (22. a ed.). Recuperado de: http://www.rae.es/rae.html

Ribes, E. (2001). Functional dimensions of social behavior: Theoretical considerations and some preliminary data. Revista Mexicana de Análisis de la Conducta, 27, 285-306.

Ribes, E. (2010). Social interactions: Conceptual reflections and an experimental approach. En R. Schwarzer \& P. A. Frensch (Eds.), Personality, human development and culture: International perspectives on psychological science, Vol. II (pp. 275288). New York, NY: Psychology Press.

Ribes, E. y López, F. (1985). Teoría de la conducta un análisis de campo y paramétrico. México, D.F.: Trillas.

Ribes, E., \& Pulido, L. (2015). Reciprocidad, tipos de contingencias sociales sistémicas y lenguaje: investigación de las interacciones interindividuales. Revista Mexicana de Psicología, 32(1), 81-91.

Ribes, E., \& Rangel, N. (2002). Choice between individual and shared contingencies in children and adults. European Journal of Behavior Analysis, 3, 61-73. 
Ribes, E., Rangel, N., Carbajal, G., \& Peña, E. (2003a). Choice between individual and shared social contingencies in children: An experimental replication in a natural setting. European Journal of Behavior Analysis, 4, 105-114.

Ribes, E., Rangel, N., Casillas, J., Álvarez, A., Gudiño, M., Zaragoza, A., \& Hernández, H. (2003b). Inequidad y asimetría de las consecuencias en la elección entre contingencias individuales y sociales. Revista Mexicana de Análisis de la Conducta, 29, 385-401.

Ribes, E., Rangel, N., Juárez, A., Contreras, S., Abreu, A., Gudiño, M., \& Casillas, J. (2003c). Respuestas "sociales" forzadas y cambio de preferencias entre contingencias individuales y sociales en niños y adultos. Acta Comportamentalia, 11, 197-234.

Ribes, E., Rangel, N., \& López, F. (2008a). Análisis teórico de las dimensiones funcionales del comportamiento social. Revista Mexicana de Psicología, 25, 1, 45-57.

Ribes, E., Rangel, N., Pulido, L., Valdez, U., Ramírez, E., Jiménez, C., \& Hernández, M. (2010). Reciprocity of responding as a determinant of partial-altruistic behavior in humans. European Journal of Behavior Analysis, 11, 105-114.

Ribes, E., Rangel, N., Ramírez, E. Valdez, U., Romero, C., \& Jiménez, C. (2008b). Verbal and-nonverbal induction of reciprocity in a partial-altruism social interaction. European Journal of Behavior Analysis, 9, 53-72.

Rousseau, J. J. (1762/2005) Emilio, o de la educación. Madrid, España: Alianza Editorial.

Sahlins, (1972). Stone Age Economics. Chicago, IL: Aldine-Atherton, Inc.

Schmitt, D. R., \& Marwell, G. (1968). Stimulus Control in the Experimental Study of Cooperation. Journal of the Experimental Analysis of Behavior, 11, 571-574.

Schmitt, D. R., \& Marwell, G. (1971a). Avoidance of Risk as a Determinant of Cooperation. Journal of the Experimental Analysis of Behavior, 16, 367- 374.

Schmitt, D. R., \& Marwell, G. (1971b). Taking and the Disruption of Cooperation. Journal of the Experimental Analysis of Behavior, 15, 405-412.

Schuster, R., \& Perelberg, A. (2004). Why cooperate? An economic perspective is not enough. Behavioural Processes, 66, 261-277.

Secord, P., \& Backman, C. (1974). Social psychology. New York, NY: McGraw-Hill.

Skinner, B. F. (1953). Science and Human Behavior. New York, NY: McMillan.

Skinner, B. F. (1978). Reflections on behaviorism and society. Englewood Cliffs, NJ: Prentice Hall.

Thibaut, J., \& Kelley, H. (1959). The social psychology of groups. New York, NY: J. Wiley.

Torche, F., \& Valenzuela, E. (2011). Trust and reciprocity: A theoretical distinction of the sources of social capital. European Journal of Social Theory, 14(2), 181-198.

Trivers, R. (1971). The evolution of reciprocal altruism. The Quarterly Review of Biology, 46, 35-57. 


\section{LIZBETH PULIDO AVALOS et al.}

52

Vuolevi, J.H.K., \& Van Lange, P.A.M. (2012). Boundaries of reciprocity: Incompleteness of information undermines cooperation. Acta Psychologica, 141, 67-72.

Weiner, H. (1977). An operant analysis of human altruistic responding. Journal of the Experimental Analysis of Behavior, 27, 515-528. 